\title{
Liouville-Type Theorems for Some Integral Systems
}

\author{
Zhengce Zhang \\ College of Science, Xi' an Jiaotong University, Xi' an, China \\ E-mail: zhangzc@mail.xjtu.edu.cn \\ Received March 20, 2010; revised May 21, 2010; accepted May 27, 2010
}

\begin{abstract}
In this paper, Liouville-type theorems of nonnegative solutions for some elliptic integral systems are considered. We use a new type of moving plane method introduced by Chen-Li-Ou. Our new ingredient is the use of Stein-Weiss inequality instead of Maximum Principle.
\end{abstract}

Keywords: Liouville Theorem, Integral System, Moving Plane Method

\section{Introduction}

In this paper we consider the nonnegative solutions of the systems of integral equations in $\mathbb{R}^{N}(N \geq 2), 0<\mu$ $<N$,

$$
\left\{\begin{array}{l}
u(x)=\int_{\mathbb{R}^{N}} \frac{u(y)^{q_{1}}+v(y)^{q_{2}}}{|x-y|^{N-\mu}} d y \\
v(x)=\int_{\mathbb{R}^{N}} \frac{v(y)^{p_{1}}+u(y)^{p_{2}}}{|x-y|^{N-\mu}} d y
\end{array}\right.
$$

and

$$
\left\{\begin{array}{l}
u(x)=\int_{\mathbb{R}^{N}} \frac{u(y)^{q_{1}} v(y)^{q_{2}}}{|x-y|^{N-\mu}} d y \\
v(x)=\int_{\mathbb{R}^{N}} \frac{v(y)^{p_{1}} u(y)^{p_{2}}}{|x-y|^{N-\mu}} d y
\end{array}\right.
$$

The integral systems are closely related to the following systems of differential equations in $\mathbb{R}^{N}$

$$
\left\{\begin{array}{l}
(-\Delta)^{\mu / 2} u=u^{q_{1}}+v^{q_{2}} \\
(-\Delta)^{\mu / 2} v=v^{p_{1}}+u^{p_{2}}
\end{array}\right.
$$

and

$$
\left\{\begin{array}{l}
(-\Delta)^{\mu / 2} u=u^{q_{1}} v^{q_{2}} \\
(-\Delta)^{\mu / 2} v=v^{p_{1}} u^{p_{2}}
\end{array}\right.
$$

In fact, every positive smooth solution of PDE (3)(or (4)) multiplied by a constant satisfies (1) (or (2)) respectively. This equivalence between integral and PDE systems can be verified as in the proof of Theorem 1 in [1]. For single equations, we refer to [2]. Here, in (3) and (4), we have used the following definition:

$$
(-\Delta)^{\mu / 2} u=\left(|\xi|^{\mu} u^{\wedge}\right)^{\vee}
$$

where $\wedge$ is the Fourier transformation and $\vee$ its inverse.

The question is to determine for which values of the exponents $p_{i}$ and $q_{i}$ the only nonnegative solution $(u, v)$ of (1) and (2) is trivial, i.e., $(u ; v)=(0,0)$. When $\mu=2$, is the case of the Emden-Fowler equation

$$
\Delta u+u^{k}=0, \quad u \geq 0 \text { in } \mathbb{R}^{N}
$$

When $1 \leq k<(N+2) /(N-2)(N>3)$, it has been proved in $[3,4]$ that the only solutions of (5) is $u=0$. In dimension $N=2$, a similar conclusion holds for $0 \leq k<\infty$. It is also well known that in the critical case, $k=(N+2)$ / $(N-2)$, problem (5) has a two-parameter family of solutions given by

$$
u(x)=\left(\frac{c}{d+|x-\bar{x}|^{2}}\right)^{\frac{N-2}{2}},
$$

where $c=[N(N-2) d]^{\frac{1}{2}}$ with $d>0$ and $\bar{x} \in \mathbb{R}^{N}$. If $p_{1}, q_{1}>1, p_{2}, q_{2} \geq 0$ and $\min \left\{p_{1}+2 p_{2}, q_{1}+2 q_{2}\right\} \leq(N+2) /$ $(N-2)$, using Pokhozhaev's second identity, Chen and $\mathrm{Lu}[5]$ have proved that the problem (4) has no positive radial solutions with $u(x)=u(|x|)$. Suppose that $p_{1}, p_{2}$, $q_{1}$ and $q_{2}$ satisfy $0 \leq p_{1}, q_{1} \leq 1, p_{2}, q_{2}>1$ and other related conditions, using the method of integral relations, Mitidieri [6] has proved that the problem (4) has no positive solutions of $C^{2}\left(\mathbb{R}^{N}\right)$. In the present paper, we study Problem (1) and Problem (2) by virtue of the moving plane method and obtain the following theorems of non- 
existence of positive solutions in the weaker regularity.

Theorem 1.1: Let $(u, v)$ be a nonnegative solution of Problem (1) and $\frac{N}{N-\mu}<p_{1}, p_{2}, q_{1}, q_{2}<\frac{N+\mu}{N-\mu}$ but not equal to $(N+\mu) /(N-\mu)$ at the same time. Suppose that $u \in L_{\text {loc }}^{\beta_{1}}\left(\mathbb{R}^{N}\right) \cap L_{\text {loc }}^{\alpha_{2}}\left(\mathbb{R}^{N}\right)$ and $v \in L_{\text {loc }}^{\beta_{2}}\left(\mathbb{R}^{N}\right) \cap L_{\text {loc }}^{\alpha_{1}}$ $\left(\mathbb{R}^{N}\right)$ with $\quad \beta_{1}=\frac{q_{1}-1}{\frac{N-\mu}{N} q_{1}-1}, \quad \beta_{2}=\frac{q_{2}-1}{\frac{N-\mu}{N} q_{2}-1}$, $\alpha_{1}=\frac{p_{1}-1}{\frac{N-\mu}{N} p_{1}-1}$ and $\alpha_{2}=\frac{p_{2}-1}{\frac{N-\mu}{N} p_{2}-1}$. Then both $u$ and $v$ are trivial.

Theorem 1.2: Let $(u, v)$ be a nonnegative solution of Problem (2) and $\frac{N}{N-\mu}<p_{1}+p_{2}, q_{1}+q_{2}<\frac{N+\mu}{N-\mu}$ but not both equal to $(N+\mu) /(N-\mu)$. Suppose that $u, v \in$ $L^{\beta}\left(\mathbb{R}^{N}\right) \cap L^{\alpha}\left(\mathbb{R}^{N}\right) \quad$ with $\beta=\frac{q_{1}+q_{2}-1}{\frac{N-\mu}{N}\left(q_{1}+q_{2}\right)-1}$ and $\alpha=\frac{p_{1}+p_{2}-1}{\frac{N-\mu}{N}\left(p_{1}+p_{2}\right)-1}$. Then both $u$ and $v$ are trivial.

Remark 1.1: In the proof of Theorem 1.1 or Theorem 1.2 , we only treat the case $\frac{N}{N-\mu}<p_{1}, p_{2}, q_{1}, q_{2}<$ $\frac{N+\mu}{N-\mu}$ or $\frac{N}{N-\mu}<p_{1}+p_{2}, q_{1}+q_{2}<\frac{N+\mu}{N-\mu} \quad$ respectively. The remaining cases can be handled in the same way. We leave this to the interested reader.

We shall need the following doubly weighted HardyLittlewood-Sololev inequality of Stein and Weiss (see, for example, [7])

$$
\left\|\int_{\mathbb{R}^{N}} V(x, y) f(y) d y\right\|_{L^{q}\left(\mathbb{R}^{N}\right)} \leq \Lambda_{\gamma, \tau, \lambda, p, N}\|f\|_{L^{p}\left(\mathbb{R}^{N}\right)}
$$

where $V(x, y)=|x|^{-\tau}|x-y|^{-\lambda}|y|^{-\gamma}, \quad 0 \leq \gamma<N / p^{\prime}, \quad 0 \leq \tau<N / q$, $1 / p+1 / p^{\prime}=1$ and $1 / p+(\gamma+\tau+\lambda) / N=1+1 / q$.

There are some related works about this paper. For $\mu=2, \quad p_{1}=q_{1}=0$ and $1<p_{2}, \quad q_{2} \leq(N+2) /(N-2)$ (but $p_{2}$ and $q_{2}$ are not both equal to $(N+2) /(N-2)$ ), Figueiredo and Felmer (see [8]) proved the similar Liouville-type theorems to Theorem 1.1 and Theorem 1.2 on nonnegative positive of $C^{2}\left(\mathbb{R}^{N}\right)$ using the moving plane method and Maximum Principle. Busca and Manásevich obtained a new result (see [9]) using the same method as in [8]. It allows $p_{2}$ and $q_{2}$ to reach regions where one of the two exponents is supercritical. In [10], Zhang, Wang and Li first introduced the Kelvin transforms and gave a different proof using the method of moving spheres. This approach was suggested in [11], while Li and Zhang had made significant simplifications in the proof of some Liouville theorems for a single equation in [12]. In this paper, by virtue of Hardy-Littlewood-Sololev inequality instead of Maximum Principle, we consider the integral systems (1) and (2) with $0<\mu<N$ and the general nonlinearities. Therefore, it is a generalization of Liouville-type theorems in $[5,6,8,9,13-15]$.

Let us emphasize that considerable attention has been drawn to Liouville-type results and existence of positive solutions for general nonlinear elliptic equations and systems, and that numerous related works are devoted to some of its variants, such as more general quasilinear operators, and domains. We refer the interested reader to [16-24], and some of the references therein.

In the following, we shall use $C$ to denote different constants which depend only on $N, \mu, q_{i}, p_{i}(i=1,2)$ and the solutions $u$ and $v$ in varying places.

\section{Kelvin-Type Transform and Proof of Theorem 1.1}

To prove Theorem 1.1, we shall use the method of moving planes. We first introduce the Kelvin-type transforms $u$ and $v$ as follows,

$$
\bar{u}(x)=|x|^{\mu-N} u\left(\frac{x}{|x|^{2}}\right) \text { and } \bar{v}(x)=|x|^{\mu-N} v\left(\frac{x}{|x|^{2}}\right)
$$

which are defined for $x \in \mathbb{R}^{N} \backslash\{0\}$. Then by elementary calculations, one verifies that (1) and (2) are transformed into the following form:

$$
\left\{\begin{array}{l}
\bar{u}(x)=\int_{\mathbb{R}^{N}}|x-y|^{\mu-N}\left(|y|^{-s_{1}} \bar{u}(y)^{q_{1}}+|y|^{-s_{2}} \bar{v}(y)^{q_{2}}\right) d y \\
\bar{v}(x)=\int_{\mathbb{R}^{N}}|x-y|^{\mu-N}\left(|y|^{-\eta_{1}} \bar{v}(y)^{p_{1}}+|y|^{-r_{2}} \bar{u}(y)^{p_{2}}\right) d y
\end{array}\right.
$$

and

$$
\left\{\begin{array}{l}
(-\Delta)^{\mu / 2} \bar{u}=|x|^{-s_{1}} \bar{u}^{q_{1}}+|x|^{-s_{2}} \bar{v}^{q_{2}} \\
(-\Delta)^{\mu / 2} \bar{v}=|x|^{-r_{1}} \bar{v}^{p_{1}}+|x|^{-r_{2}} \bar{u}^{p_{2}}
\end{array}\right.
$$

with $s_{i}=(N+\mu)-(N-\mu) q_{i}>0, \quad r_{i}=(N+\mu)-(N-\mu)$ $p_{i}>0(i=1,2)$, and

$$
\left\{\begin{array}{l}
\bar{u}(x)=\int_{\mathbb{R}^{N}}|x-y|^{\mu-N}|y|^{-s} \bar{u}(y)^{q_{1}} \bar{v}(y)^{q_{2}} d y \\
\bar{v}(x)=\int_{\mathbb{R}^{N}}|x-y|^{\mu-N}|y|^{-r} \bar{v}(y)^{p_{1}} \bar{u}(y)^{p_{2}} d y
\end{array}\right.
$$


and

$$
\left\{\begin{array}{l}
(-\Delta)^{\mu / 2} \bar{u}=|x|^{-s} \bar{u}^{q_{1}} \bar{v}^{q_{2}} \\
(-\Delta)^{\mu / 2} \bar{v}=|x|^{-r} \bar{v}^{p_{1}} \bar{u}^{p_{2}}
\end{array}\right.
$$

where $s=(N+\mu)-(N-\mu)\left(q_{1}+q_{2}\right)>0$ and $r=(N+\mu)-$ $(N-\mu)\left(p_{1}+p_{2}\right)>0$. Obviously, both $\bar{u}(x)$ and $\bar{v}(x)$ have singularities at origin. Since $u$ is locally $L^{\beta_{1}} \cap L^{\alpha_{2}}$ and $v$ is locally $L^{\beta_{2}} \cap L^{\alpha_{1}}$ (in Theorem 1.1), it is easy to see that $\bar{u}(x)$ and $\bar{v}(x)$ have no singularity at infinity, i.e., for any domain $\Omega$ that is a positive distance away from the origin,

$$
\begin{gathered}
\int_{\Omega} \bar{u}^{\beta_{1}}(y) d y<\infty \text { and } \int_{\Omega} \bar{u}^{\alpha_{2}}(y) d y<\infty . \\
\int_{\Omega} \bar{v}^{\beta_{2}}(y) d y<\infty \text { and } \int_{\Omega} \bar{v}^{\alpha_{1}}(y) d y<\infty .
\end{gathered}
$$

For $\lambda \in \mathbb{R}$, define

$$
\sum_{\lambda}=\left\{x=\left(x_{1}, x_{2} \cdots, x_{N}\right) \mid x_{1} \geq \lambda\right\} .
$$

Let $x^{\lambda}=\left\{2 \lambda-x_{1}, x_{2}, \cdots x_{N}\right\}$ and define

$$
\bar{u}_{\lambda}(x)=\bar{u}\left(x^{\lambda}\right) \text { and } \bar{v}_{\lambda}(x)=\bar{v}\left(x^{\lambda}\right)
$$

Lemma 2.1: For any solution $(\bar{u}, \bar{v})$ of $(8)$, we have

$$
\begin{aligned}
& \bar{u}_{\lambda}(x)-\bar{u}(x)=\int_{\sum_{\lambda}}\left(\frac{1}{|x-y|^{N-\mu}}-\frac{1}{\left|x^{\lambda}-y\right|^{N-\mu}}\right) \\
& \cdot\left(\frac{1}{\left|y^{\lambda}\right|^{s_{1}}} \bar{u}_{\lambda}^{q_{1}}-\frac{1}{|y|^{s_{1}}} \bar{u}^{q_{1}}\right) d y+\int_{\sum_{\lambda}}\left(\frac{1}{|x-y|^{N-\mu}}\right. \\
& \left.-\frac{1}{\left|x^{\lambda}-y\right|^{N-\mu}}\right)\left(\frac{1}{\left|y^{\lambda}\right|^{s_{2}}} \bar{v}_{\lambda}^{q_{2}}-\frac{1}{|y|^{s_{2}}} \bar{v}^{q_{2}}\right) d y
\end{aligned}
$$

and

$$
\begin{aligned}
& \bar{v}_{\lambda}(x)-\bar{v}(x)=\int_{\sum_{\lambda}}\left(\frac{1}{|x-y|^{N-\mu}}-\frac{1}{\left|x^{\lambda}-y\right|^{N-\mu}}\right) \\
& \cdot\left(\frac{1}{\left|y^{\lambda}\right|^{r_{1}}} \bar{v}_{\lambda}^{p_{1}}-\frac{1}{|y|^{r_{1}}} \bar{v}^{p_{1}}\right) d y+\int_{\sum_{\lambda}}\left(\frac{1}{|x-y|^{N-\mu}}\right. \\
& \left.-\frac{1}{\left|x^{\lambda}-y\right|^{N-\mu}}\right)\left(\frac{1}{\left|y^{\lambda}\right|^{r_{2}}} \bar{u}_{\lambda}^{p_{2}}-\frac{1}{|y|^{r_{2}}} \bar{u}^{p_{2}}\right) d y
\end{aligned}
$$

Proof. Let $\sum_{\lambda}^{c}=\left\{x=\left(x_{1}, \cdots x_{N}\right) \mid x_{1}<\lambda\right\}$. Then it is easy to see that

$$
\begin{aligned}
\bar{u}(x)= & \int_{\sum_{\lambda}} \frac{1}{|x-y|^{N-\mu}} \frac{1}{|y|^{s_{1}}} \bar{u}(y)^{q_{1}} d y \\
& +\int_{\sum_{\lambda}} \frac{1}{|x-y|^{N-\mu}} \frac{1}{|y|^{s_{2}}} \bar{v}(y)^{q_{2}} d y
\end{aligned}
$$

$$
\begin{aligned}
& +\int_{\sum_{\lambda}^{c}} \frac{1}{|x-y|^{N-\mu}} \frac{1}{|y|^{s_{1}}} \bar{u}(y)^{q_{1}} d y \\
& +\int_{\sum_{\lambda}^{c}} \frac{1}{|x-y|^{N-\mu}} \frac{1}{|y|^{s_{2}}} \bar{v}(y)^{q_{2}} \\
& =\int_{\sum_{\lambda}} \frac{1}{|x-y|^{N-\mu}} \frac{1}{|y|^{s_{1}}} \bar{u}(y)^{q_{1}} d y \\
& +\int_{\sum \lambda} \frac{1}{|x-y|^{N-\mu}} \frac{1}{|y|^{s_{2}}} \bar{v}(y)^{q_{2}} d y \\
& +\int_{\sum_{\lambda}} \frac{1}{\left|x-y^{\lambda}\right|^{N-\mu}} \frac{1}{\left|y^{\lambda}\right|^{s_{1}}} \bar{u}\left(y^{\lambda}\right)^{q_{1}} d y \\
& +\int_{\sum_{\lambda}} \frac{1}{\left|x-y^{\lambda}\right|^{N-\mu}} \frac{1}{\left|y^{\lambda}\right|^{s_{2}}} \bar{v}\left(y^{\lambda}\right)^{q_{2}} \\
& =\int_{\sum_{\lambda}} \frac{1}{|x-y|^{N-\mu}} \frac{1}{|y|^{s_{1}}} \bar{u}(y)^{q_{1}} d y \\
& +\int_{\sum_{\lambda}} \frac{1}{|x-y|^{N-\mu}} \frac{1}{|y|^{s_{2}}} \bar{v}(y)^{q_{2}} d y \\
& +\int_{\sum_{\lambda}} \frac{1}{\left|x^{\lambda}-y\right|^{N-\mu}} \frac{1}{\left|y^{\lambda}\right|^{s_{1}}} \bar{u}_{\lambda}(y)^{q_{1}} d y \\
& +\int_{\Sigma_{\lambda}} \frac{1}{\left|x^{\lambda}-y\right|^{N-\mu}} \frac{1}{\left|y^{\lambda}\right|^{s_{2}}} \bar{v}_{\lambda}(y)^{q_{2}}
\end{aligned}
$$

Here we have used the fact that $\left|x-y^{\lambda}\right|=\left|x^{\lambda}-y\right|$.

Substituting $x$ by $x^{\lambda}$, we get

$$
\begin{aligned}
\bar{u}\left(x^{\lambda}\right)= & \int_{\sum_{\lambda}} \frac{1}{\left|x^{\lambda}-y\right|^{N-\mu}} \frac{1}{|y|^{s_{1}}} \bar{u}(y)^{q_{1}} d y \\
& +\int_{\sum_{\lambda}} \frac{1}{\left|x^{\lambda}-y\right|^{N-\mu}} \frac{1}{|y|^{s_{2}}} \bar{v}(y)^{q_{2}} d y \\
& +\int_{\sum_{\lambda}} \frac{1}{|x-y|^{N-\mu}} \frac{1}{\left|y^{\lambda}\right|^{s_{1}}} \bar{u}_{\lambda}(y)^{q_{1}} d y \\
& +\int_{\sum_{\lambda}} \frac{1}{|x-y|^{N-\mu}} \frac{1}{\left|y^{\lambda}\right|^{s_{2}}} \bar{v}_{\lambda}(y)^{q_{2}}
\end{aligned}
$$

Thus

$$
\begin{aligned}
& \bar{u}_{\lambda}(x)-\bar{u}(x)=\int_{\sum_{\lambda}}\left(\frac{1}{|x-y|^{N-\mu}}-\frac{1}{\left|x^{\lambda}-y\right|^{N-\mu}}\right) \\
& \cdot\left(\frac{1}{\left|y^{\lambda}\right|^{s_{1}}} \bar{u}_{\lambda}^{q_{1}}-\frac{1}{|y|^{s_{1}}} \bar{u}^{q_{1}}\right) d y+\int_{\sum_{\lambda}}\left(\frac{1}{|x-y|^{N-\mu}}\right. \\
& \left.-\frac{1}{\left|x^{\lambda}-y\right|^{N-\mu}}\right)\left(\frac{1}{\left|y^{\lambda}\right|^{s_{2}}} \bar{v}_{\lambda}^{q_{2}}-\frac{1}{|y|^{s_{2}}} \bar{v}^{q_{2}}\right) d y
\end{aligned}
$$


This implies (14). Similarly, we can get (15). So Lemma 2.1 is proved.

Proof of Theorem 1.1.

Outline: Let $x_{1}$ and $x_{2}$ be any two points in $\mathbb{R}^{N}$. We shall show that

$$
u\left(x_{1}\right)=u\left(x_{2}\right) \text { and } v\left(x_{1}\right)=v\left(x_{2}\right)
$$

and therefore $u$ and $v$ must be constants. This is impossible unless $u=v=0$. To obtain this, we show that $u$ and $v$ are symmetric about the midpoint $\left(x_{1}+x_{2}\right) / 2$. Since the integral equations are invariant under translation, we may assume that the midpoint is at the origin. Let $\bar{u}$ and $\bar{v}$ be the Kelvin-type transformations of $u$ and $v$ respectively. Then what left to prove is that $u$ and $v$ are symmetric about the origin. We shall carry this out in the following three steps.

Step 1. Define

$$
\sum_{\lambda}^{\bar{u}}=\left\{x \mid x \in \sum_{\lambda}, \bar{u}(x)<\bar{u}_{\lambda}(x)\right\}
$$

and

$$
\sum_{\lambda}^{\bar{v}}=\left\{x \mid x \in \sum_{\lambda}, \bar{v}(x)<\bar{v}_{\lambda}(x)\right\}
$$

We want to show that for sufficiently negative values of $\lambda$, both $\sum_{\lambda}^{\bar{u}}$ and $\sum_{\lambda}^{\bar{v}}$, must be empty. We note that $|x-y| \leq\left|x^{\lambda}-y\right|, \quad \forall x, \quad y \in \sum_{\lambda}$. Moreover, since $\lambda<$ $0,\left|y^{\lambda}\right| \geq|y|$ for any $y \in \sum_{\lambda}$. Then by (14), for any $x \in \sum_{\lambda}^{\bar{u}}$,

$$
\begin{aligned}
& \bar{u}_{\lambda}(x)-\bar{u}(x) \\
\leq & \int_{\sum_{\lambda}}\left(|x-y|^{\mu-N}-\left|x^{\lambda}-y\right|^{\mu-N}\right) \\
& \cdot|y|^{-s_{1}}\left(\bar{u}_{\lambda}(y)^{q_{1}}-\bar{u}(y)^{q_{1}}\right) d y \\
& +\int_{\sum_{\lambda}}\left(|x-y|^{\mu-N}-\left|x^{\lambda}-y\right|^{\mu-N}\right) \\
& \cdot|y|^{-s_{2}}\left(\bar{v}_{\lambda}(y)^{q_{2}}-\bar{v}(y)^{q_{2}}\right) d y \\
\leq & \int_{\sum_{\lambda}^{\bar{u}}}|x-y|^{\mu-N}|y|^{-s_{1}}\left(\bar{u}_{\lambda}(y)^{q_{1}}-\bar{u}(y)^{q_{1}}\right) d y \\
& +\int_{\sum_{\bar{\nu}}^{\bar{v}}}|x-y|^{\mu-N}|y|^{-s_{2}}\left(\bar{v}_{\lambda}(y)^{q_{2}}-\bar{v}(y)^{q_{2}}\right) d y \\
\leq & q_{1} \int_{\sum_{\lambda}^{\bar{u}}}|x-y|^{\mu-N}|y|^{-s_{1}}\left[\bar{u}_{\lambda}^{q_{1}-1}\left(\bar{u}_{\lambda}-\bar{u}\right)\right](y) d y \\
& +q_{2} \int_{\sum_{\lambda}^{\bar{v}}}|x-y|^{\mu-N}|y|^{-s_{2}}\left[\bar{v}_{\lambda}^{q_{2}-1}\left(\overline{v_{\lambda}}-\bar{v}\right)\right](y) d y
\end{aligned}
$$

It follows from (7) and then the Hölder inequality that, for any $\gamma>\max \left\{N /\left(N+s_{1}-\mu\right), N /\left(N+s_{2}-\mu\right)\right\}$,

$$
\begin{aligned}
& \left\|\bar{u}_{\lambda}-\bar{u}\right\|_{L^{\gamma}\left(\sum_{\lambda}^{\bar{u}}\right)} \\
\leq & C\left(\| \int_{\sum_{\lambda}^{\bar{u}}}|x-y|^{\mu-N}|y|^{-s_{1}}\right. \\
\cdot & {\left[\bar{u}_{\lambda}^{q_{1}-1}\left(\bar{u}_{\lambda}-\bar{u}\right)\right](y) d y \|_{L^{\gamma}\left(\sum_{\lambda}^{\bar{u}}\right)} }
\end{aligned}
$$

$$
\begin{array}{r}
+\| \int_{\sum_{\lambda}^{\bar{v}}}|x-y|^{\mu-N}|y|^{-s_{2}} \\
\left.\cdot\left[\bar{v}_{\lambda}^{q_{2}-1}\left(\bar{v}_{\lambda}-\bar{v}\right)\right](y) d y \|_{L^{\gamma}\left(\sum_{\lambda}^{\bar{u}}\right)}\right) \\
\leq C\left(\left\|\bar{u}_{\lambda}\right\|_{L^{\beta_{1}}\left(\sum_{\lambda}^{\bar{u}}\right)}^{q_{1}-1}\left\|\bar{u}_{\lambda}-\bar{u}\right\|_{L^{\gamma}\left(\sum_{\lambda}^{\bar{u}}\right)}\right. \\
\left.+\left\|\bar{v}_{\lambda}\right\|_{L^{\beta_{2}}\left(\sum_{\lambda}^{\bar{\nu}}\right)}^{q_{2}-1}\left\|\bar{v}_{\lambda}-\bar{v}\right\|_{L^{\gamma}\left(\sum_{\lambda}^{\bar{v}}\right)}\right)
\end{array}
$$

where $\beta_{1}=\frac{q_{1}-1}{\frac{N-\mu}{N} q_{1}-1}$ and $\beta_{2}=\frac{q_{2}-1}{\frac{N-\mu}{N} q_{2}-1}$.

Similarly, one can show that

$$
\begin{gathered}
\left\|\bar{v}_{\lambda}-\bar{v}\right\|_{L^{\gamma}\left(\sum_{\lambda}^{\bar{v}}\right)} \\
\leq C\left(\left\|\bar{v}_{\lambda}\right\|_{L^{\alpha_{1}}\left(\sum_{\lambda}^{\bar{v}}\right)}^{p_{1}-1}\left\|\bar{v}_{\lambda}-\bar{v}\right\|_{L^{\gamma}\left(\sum_{\lambda}^{\bar{v}}\right)}\right. \\
\left.+\left\|\bar{u}_{\lambda}\right\|_{L^{\alpha_{2}}\left(\sum_{\lambda}^{\bar{u}}\right)}^{p_{2}-1}\left\|\bar{u}_{\lambda}-\bar{u}\right\|_{L^{\gamma}\left(\sum_{\lambda}^{\bar{u}}\right)}\right)
\end{gathered}
$$

where $\alpha_{1}=\frac{p_{1}-1}{\frac{N-\mu}{N} p_{1}-1}$ and $\alpha_{2}=\frac{p_{2}-1}{\frac{N-\mu}{N} p_{2}-1}$.

Combining (16) and (17), we have

$$
\begin{aligned}
& \left\|\bar{u}_{\lambda}-\bar{u}\right\|_{L^{\gamma}\left(\sum_{\lambda}^{\bar{u}}\right)} \\
\leq & C\left[\left(1-C\left\|\bar{u}_{\lambda}\right\|_{L^{\beta_{1}}\left(\sum_{\lambda}^{\bar{u}}\right)}^{q_{1}-1}\right)\right]^{-1}\left\|\bar{u}_{\lambda}\right\|_{L^{\alpha_{2}}\left(\sum_{\lambda}^{\bar{u}}\right)}^{p_{2}-1} \\
\cdot & {\left[\left(1-C\left\|_{\bar{v}_{\lambda}}\right\|_{L^{\alpha_{1}}\left(\sum_{\lambda}^{\bar{v}}\right)}^{p_{1}-1}\right)\right]^{-1}\left\|\bar{v}_{\lambda}\right\|_{L^{\beta_{2}}\left(\sum_{\lambda}^{\bar{v}}\right)}^{q_{2}-1} } \\
\cdot & \left\|\bar{u}_{\lambda}-\bar{u}\right\|_{L^{\gamma}\left(\sum_{\lambda}^{\bar{u}}\right)}
\end{aligned}
$$

By conditions (12) and (13), we can choose $N$ sufficiently large, such that for $\lambda \leq-N$, we have

$$
\begin{gathered}
C\left[\left(1-C\left\|\bar{u}_{\lambda}\right\|_{L^{\beta_{1}}\left(\sum_{\lambda}^{\bar{u}}\right)}^{q_{1}-1}\right)\right]^{-1}\left\|\bar{u}_{\lambda}\right\|_{L^{\alpha_{2}}\left(\sum_{\lambda}^{\bar{u}}\right)}^{p_{2}-1} \\
\cdot\left[\left(1-C\left\|\bar{v}_{\lambda}\right\|_{L^{\alpha_{1}}\left(\sum_{\lambda}^{\bar{v}}\right)}^{p_{1}-1}\right)\right]^{-1}\left\|\bar{v}_{\lambda}\right\|_{L^{\beta_{2}}\left(\sum_{\lambda}^{\bar{v}}\right)}^{q_{2}-1} \leq \frac{1}{2} .
\end{gathered}
$$

Then (18) implies that

$$
\left\|\bar{u}_{\lambda}-\bar{u}\right\|_{L^{\gamma}\left(\sum_{\lambda}^{\bar{u}}\right)}=0
$$

and therefore $\sum_{\lambda}^{\bar{u}}$ must be measure zero, and hence empty. Similarly, one can show that $\sum_{\lambda}^{\bar{v}}$ is empty.

Step 2. Now we have that for $\lambda \leq-N$,

$$
\bar{u}(x) \geq \bar{u}_{\lambda}(x) \text { and } \bar{v}(x) \geq \bar{v}_{\lambda}(x) \quad \forall x \in \sum_{\lambda} .
$$

So the plane can start moving continuously from $\lambda \leq-N$ to the right as long as (19) holds. Suppose that there exists $\lambda_{0}<0$ such that, on $\sum_{\lambda_{0}}$,

$$
\bar{u}(x) \geq \bar{u}_{\lambda_{0}}(x) \text { and } \bar{v}(x) \geq \bar{v}_{\lambda_{0}}(x),
$$


but meas $\left\{x \in \sum_{\lambda_{0}} \mid \bar{u}(x)>\bar{u}_{\lambda_{0}}(x)\right\}>0$ or meas $\left\{x \in \sum_{\lambda_{0}}\right.$ $\left.\mid \bar{v}(x)>\bar{v}_{\lambda_{0}}(x)\right\}>0$.

We shall show that the plane can move further to the right, i.e., there exists an $\varepsilon$ depending on $N, p_{i}, q_{i}(i=1$, $2)$, and the solutions $(\bar{u}, \bar{v})$ such that (19) holds for all $\lambda \in\left[\lambda_{0}, \lambda_{0}+\varepsilon\right)$.

Once either meas $\left\{x \in \sum_{\lambda_{0}} \mid \bar{u}(x)>\bar{u}_{\lambda_{0}}(x)\right\}>0$ or meas $\left\{x \in \sum_{\lambda_{0}} \mid \bar{v}(x)>\bar{v}_{\lambda_{0}}(x)\right\}>0$ holds, we see by (14) that in $\bar{u}(x)>\bar{u}_{\lambda_{0}}(x)$ the interior of $\sum_{\lambda_{0}}$. Let

$$
\widetilde{\sum_{\lambda_{0}}^{\bar{u}_{0}}}=\left\{x \mid x \in \sum_{\lambda_{0}}, \bar{u}(x) \leq \bar{u}_{\lambda_{0}}(x)\right\}
$$

and

$$
\widetilde{\sum_{\lambda_{0}}^{\bar{v}}}=\left\{x \mid x \in \sum_{\lambda_{0}}, \bar{v}(x) \leq \bar{v}_{\lambda_{0}}(x)\right\} .
$$

It is easy to see that $\widetilde{\sum \bar{u}_{0}}$ has measure zero, and $\lim _{\lambda \rightarrow \lambda_{0}} \sum \bar{u}_{\lambda_{0}} \subset \sum_{\lambda_{0}}^{\bar{u}_{0}}$. The similar results also hold for $v$. Let $\Omega^{*}$ be the reflection of the set $\Omega$ about the plane $x_{1}=\lambda$. From (16) and (17), we deduce

$$
\begin{aligned}
& \left\|\bar{u}_{\lambda}-\bar{u}\right\|_{L^{\gamma}\left(\sum_{\lambda}^{\bar{u}}\right)} \\
\leq & C\left[\left(1-C\left\|\bar{u}_{\lambda}\right\|_{L^{\beta_{1}}\left(\left(\sum_{\lambda}^{\bar{u}}\right)^{*}\right)}^{q_{1}-1}\right)\right]^{-1}\left\|\bar{u}_{\lambda}\right\|_{L^{\alpha_{2}}\left(\left(\sum_{\lambda}^{\bar{u}}\right)^{*}\right)}^{p^{2}-1} \\
\cdot & {\left[\left(1-C\left\|\bar{v}_{\lambda}\right\|_{L^{\alpha_{1}}\left(\left(\sum_{\lambda}^{\bar{\nu}}\right)^{*}\right)}^{p_{1}-1}\right)\right]^{-1}\left\|\bar{v}_{\lambda}\right\|_{L^{\beta_{2}}\left(\left(\sum_{\lambda}^{\bar{\nu}}\right)^{*}\right)}^{q_{2}-1} } \\
\cdot & \left\|\bar{u}_{\lambda}-\bar{u}\right\|_{L^{\gamma}\left(\sum_{\lambda}^{\bar{u}}\right)}
\end{aligned}
$$

Again conditions (12) and (13) ensure that one can choose $\varepsilon$ small enough such that, for all $\lambda \in$ $\left[\lambda_{0}, \lambda_{0}+\varepsilon\right)$,

$$
\begin{gathered}
C\left[\left(1-C\left\|\bar{u}_{\lambda}\right\|_{L^{\beta_{1}}\left(\left(\sum_{\lambda}^{\bar{u}}\right)^{*}\right)}^{q_{1}-1}\right)\right]^{-1}\left\|\bar{u}_{\lambda}\right\|_{L^{\alpha_{2}}\left(\left(\sum_{\lambda}^{\bar{u}}\right)^{*}\right)}^{p_{2}-1} \\
\cdot\left[\left(1-C\left\|\bar{v}_{\lambda}\right\|_{L^{\alpha_{1}}\left(\left(\sum_{\bar{\nu}}^{\bar{\nu}}\right)^{*}\right)}^{p_{1}-1}\right)\right]^{-1}\left\|\bar{v}_{\lambda}\right\|_{L^{\beta_{2}}\left(\left(\sum_{\bar{\nu}}^{\bar{\nu}}\right)^{*}\right)}^{q_{2}-1} \leq \frac{1}{2} .
\end{gathered}
$$

Now by (20), we have

$$
\left\|\bar{u}_{\lambda}-\bar{u}\right\|_{L^{\gamma}\left(\sum_{\lambda}^{\bar{u}}\right)}=0
$$

and therefore $\sum_{\lambda}^{\bar{u}}$ must be empty. Similarly, $\sum_{\lambda}^{\bar{v}}$ must be empty, too.

Step 3. If the plane stops at $\lambda_{0}<0$, then $\bar{u}(x)$ and $\bar{v}(x)$ must be symmetric and monotone about the plane $x_{1}=\lambda_{0}$. This implies that $\bar{u}(x)$ and $\bar{v}(x)$ have no singularity at the origin. But Equation (8) tells us that this is impossible if $\bar{u}(x)$ and $\bar{v}(x)$ are nontrivial. Hence we can move the plane to $x_{1}=0$. Then $\bar{u}(x)$ and $\bar{v}(x)$ must be symmetric about the origin. Thus $u=v=$ 0 . This completes the proof.

\section{Proof of Theorem 1.2}

In this section we establish Theorem 1.2. The proof is along the same line of the proof of Theorem 1.1. First establishing Lemma 3.1 which is similar to Lemma 2.1, we omit its proof.

Lemma 3.1: For any solution $(\bar{u}, \bar{v})$ of $(10)$, we have

$$
\begin{aligned}
\bar{u}_{\lambda}(x)-\bar{u}(x)= & \int_{\sum_{\lambda}}\left(\frac{1}{|x-y|^{N-\mu}}-\frac{1}{\left|x^{\lambda}-y\right|^{N-\mu}}\right) \\
& \cdot\left(\frac{1}{\left|y^{\lambda}\right|^{s}} \bar{u}_{\lambda}^{q_{1}} \bar{v}_{\lambda}^{q_{2}}-\frac{1}{|y|^{s}} \bar{u}^{q_{1}} \bar{v}^{q_{2}}\right) d y
\end{aligned}
$$

and

$$
\begin{aligned}
\bar{v}_{\lambda}(x)-\bar{v}(x)= & \int_{\sum_{\lambda}}\left(\frac{1}{|x-y|^{N-\mu}}-\frac{1}{\left|x^{\lambda}-y\right|^{N-\mu}}\right) \\
& \cdot\left(\frac{1}{\left|y^{\lambda}\right|^{r}} \bar{v}_{\lambda}^{p_{1}} \bar{u}_{\lambda}^{p_{2}}-\frac{1}{|y|^{r}} \bar{v}^{p_{1}} \bar{u}^{p_{2}}\right) d y
\end{aligned}
$$

\section{Proof of Theorem 1.2.}

Here we only provide necessary changes in Step 1, the rest is same as the proof of Theorem 1.1. $\sum_{\lambda}^{\bar{u}}$ and $\sum_{\lambda}^{\bar{v}}$ are defined as above. We want to show that for sufficiently negative values of $\lambda$, both $\sum_{\lambda}^{\bar{u}}$ and $\sum_{\lambda}^{\bar{v}}$ must be empty. We note that $|x-y| \leq\left|x^{\lambda}-y\right| \quad \forall x, \quad y \in \sum_{\lambda}$. Moreover, since $\lambda<0,\left|y^{\lambda}\right| \geq|y|$ for any $y \in \sum_{\lambda}$. Then by (21), for any $x \in \sum_{\lambda}^{\bar{u}}$,

$$
\begin{aligned}
& \bar{u}_{\lambda}(x)-\bar{u}(x) \\
\leq & \int_{\sum_{\lambda}}\left(|x-y|^{\mu-N}-\left|x^{\lambda}-y\right|^{\mu-N}\right)|y|^{-s} \\
& \cdot\left[\bar{u}_{\lambda}(y)^{q_{1}} \bar{v}_{\lambda}(y)^{q_{2}}-\bar{u}(y)^{q_{1}} \bar{v}(y)^{q_{2}}\right] d y \\
\leq & \int_{\sum_{\lambda}^{\bar{u}}}|x-y|^{\mu-N}|y|^{-s} \bar{v}(y)^{q_{2}}\left[\bar{u}_{\lambda}(y)^{q_{1}}-\bar{u}(y)^{q_{1}}\right] d y \\
& +\int_{\sum_{\lambda}^{\bar{\nu}}}|x-y|^{\mu-N}|y|^{-s} \bar{u}_{\lambda}(y)^{q_{1}}\left[\bar{v}_{\lambda}(y)^{q_{2}}-\bar{v}(y)^{q_{2}}\right] d y \\
\leq & q_{1} \int_{\sum_{\lambda}^{\bar{u}}}|x-y|^{\mu-N}|y|^{-s}\left[\bar{u}_{\lambda}^{q_{1}-1} \bar{v}^{q_{2}}\left(\bar{u}_{\lambda}-\bar{u}\right)\right](y) d y \\
& +q_{2} \int_{\sum_{\lambda}^{\bar{\nu}}}|x-y|^{\mu-N}|y|^{-s}\left[\bar{v}_{\lambda}^{q_{2}-1} \bar{u}_{\lambda}^{q_{1}}\left(\bar{v}_{\lambda}-\bar{v}\right)\right](y) d y .
\end{aligned}
$$

It follows from (7) and then the Hölder inequality that, for any $\gamma>N /(N+s-\mu)$, 


$$
\begin{gathered}
\left\|\bar{u}_{\lambda}-\bar{u}\right\|_{L^{\gamma}\left(\sum_{\lambda}^{\bar{u}}\right)} \\
\leq C\left(\| \int_{\sum_{\lambda}^{\bar{u}}}|x-y|^{\mu-N}|y|^{-s}\right. \\
\cdot\left[\bar{u}_{\lambda}^{q_{1}-1} \bar{v}^{q_{2}}\left(\bar{u}_{\lambda}-\bar{u}\right)\right](y) d y \|_{L^{\gamma}\left(\sum_{\lambda}^{\bar{u}}\right)} \\
+\| \int_{\sum_{\lambda}^{\bar{v}}}|x-y|^{\mu-N}|y|^{-s} \\
\left.\cdot\left[\bar{v}_{\lambda}^{q_{2}-1} \bar{u}_{\lambda}^{q_{1}}\left(\bar{v}_{\lambda}-\bar{v}\right)\right](y) d y \|_{L^{\gamma}\left(\sum_{\lambda}^{\bar{u}}\right)}\right) \\
C\left(\left\|\bar{u}_{\lambda}\right\|_{L^{\beta}\left(\sum_{\lambda}^{\bar{u}}\right)}^{q_{1}-1}\|\bar{v}\|_{L^{\beta}\left(\sum_{\lambda}^{\bar{u}}\right)}^{q_{2}}\left\|\bar{u}_{\lambda}-\bar{u}\right\|_{L^{\gamma}\left(\sum_{\lambda}^{\bar{u}}\right)}\right. \\
\left.+\left\|\bar{u}_{\lambda}\right\|_{L^{\beta}\left(\sum_{\lambda}^{\bar{\nu}}\right)}^{q_{1}}\left\|\bar{v}_{\lambda}\right\|_{L^{\beta}\left(\sum_{\lambda}^{\bar{u}}\right)}^{q_{2}-1}\left\|\bar{v}_{\lambda}-\bar{v}\right\|_{L^{\gamma}\left(\sum_{\lambda}^{\bar{\nu}}\right)}\right)
\end{gathered}
$$

Similarly, one can show that

$$
\begin{aligned}
& \left\|\bar{v}_{\lambda}-\bar{v}\right\|_{L^{\gamma}\left(\sum_{\lambda}^{\bar{v}}\right)} \\
& \leq C\left(\left\|\bar{v}_{\lambda}\right\|_{L^{\alpha}\left(\sum_{\lambda}^{\bar{u}}\right)}^{p_{1}-1}\left\|\bar{u}_{\lambda}\right\|_{L^{\alpha}\left(\sum_{\lambda}^{\bar{u}}\right)}^{p_{2}-1}\left\|\bar{u}_{\lambda}-\bar{u}\right\|_{L^{\gamma}\left(\sum_{\lambda}^{\bar{u}}\right)}\right. \\
& \left.\quad+\left\|\bar{v}_{\lambda}\right\|_{L^{\alpha}\left(\sum_{\lambda}^{\bar{v}}\right)}^{p_{1}-1}\|\bar{u}\|_{L^{\alpha}\left(\sum_{\lambda}^{\bar{v}}\right)}^{p_{2}}\left\|\bar{v}_{\lambda}-\bar{v}\right\|_{L^{\gamma}\left(\sum_{\lambda}^{\bar{v}}\right)}\right)
\end{aligned}
$$

where $\alpha=\frac{p_{1}+p_{2}-1}{\frac{N-\mu}{N}\left(p_{1}+p_{2}\right)-1}$ and $\beta=\frac{q_{1}+q_{2}-1}{\frac{N-\mu}{N}\left(q_{1}+q_{2}\right)-1}$.

Combining (23) and (24), we have

$$
\begin{aligned}
\left\|\bar{u}_{\lambda}-\bar{u}\right\|_{L^{\gamma}\left(\sum_{\lambda}^{\bar{u}}\right)} & \\
\leq C & {\left[\left(1-C\left\|\bar{u}_{\lambda}\right\|_{L^{\beta}\left(\sum_{\lambda}^{\bar{u}}\right)}^{q_{1}-1}\|\bar{v}\|_{L^{\beta}\left(\sum_{\lambda}^{\bar{u}}\right)}^{q_{2}}\right]^{-1}\right.} \\
\cdot & \left\|\bar{u}_{\lambda}\right\|_{L^{\beta}\left(\sum_{\lambda}^{\bar{\nu}}\right)}^{q_{1}}\left\|\bar{v}_{\lambda}\right\|_{L^{\beta}\left(\sum_{\lambda}^{\bar{\nu}}\right)}^{q_{\lambda}-1} \\
\cdot & {\left[\left(1-C\left\|\bar{v}_{\lambda}\right\|_{L^{\alpha}\left(\sum_{\lambda}^{\bar{\nu}}\right)}^{p_{1}-1}\|\bar{u}\|_{L^{\alpha}\left(\sum_{\lambda}^{\bar{\nu}}\right)}^{p_{2}}\right]^{-1}\right.} \\
\cdot & \left\|\bar{v}_{\lambda}\right\|_{L^{\alpha}\left(\sum_{\lambda}^{\bar{u}}\right)}^{p_{1}}\left\|\bar{u}_{\lambda}\right\|_{L^{\alpha}\left(\sum_{\lambda}^{p_{\lambda}}\right)}^{p_{2}-1} \cdot\left\|\bar{u}_{\lambda}-\bar{u}\right\|_{L^{\gamma}\left(\sum_{\lambda}^{\bar{u}}\right)}
\end{aligned}
$$

By the hypotheses in Theorem 1.2, we can choose $N$ sufficiently large, such that for $\lambda \leq-N$,

$$
\begin{gathered}
C\left[\left(1-C\left\|\bar{u}_{\lambda}\right\|_{L^{\beta}\left(\sum_{\lambda}^{\bar{u}}\right)}^{q_{1}-1}\|\bar{v}\|_{L^{\beta}\left(\sum_{\lambda}^{\bar{u}}\right)}^{q_{2}}\right]^{-1}\right. \\
\cdot\left\|\bar{u}_{\lambda}\right\|_{L^{\beta}\left(\sum_{\lambda}^{\bar{v}}\right)}^{q_{1}}\left\|\bar{v}_{\lambda}\right\|_{L^{\beta}\left(\sum_{\lambda}^{\bar{v}}\right)}^{q_{2}-1} \\
\cdot\left[\left(1-C\left\|\bar{v}_{\lambda}\right\|_{L^{\alpha}\left(\sum_{\lambda}^{\bar{v}}\right)}^{p_{1}-1}\|\bar{u}\|_{L^{\alpha}\left(\sum_{\lambda}^{\bar{v}}\right)}^{p_{2}}\right]^{-1}\right. \\
\cdot\left\|\bar{v}_{\lambda}\right\|_{L^{\alpha}\left(\sum_{\lambda}^{\bar{u}}\right)}^{p_{1}}\left\|\bar{u}_{\lambda}\right\|_{L^{\alpha}\left(\sum_{\lambda}^{\bar{u}}\right)}^{p_{2}-1} \leq \frac{1}{2} .
\end{gathered}
$$

Then (25) implies that

$$
\left\|\bar{u}_{\lambda}-\bar{u}\right\|_{L^{\gamma}\left(\sum_{\lambda}^{\bar{u}}\right)}=0
$$

and therefore $\sum_{\lambda}^{\bar{u}}$ must be measure zero, and hence empty. Similarly $\sum_{\lambda}^{\bar{v}}$ is also empty.

\section{Acknowledgements}

This work is supported by Youth Foundation of NSFC (No. 10701061), and it was completed while the author was visiting University of Notre Dame.

\section{References}

[1] W. X. Chen and C. M. Li, "Classification of Positive Solutions for Nonlinear Differential and Integral Systems with Critical Exponents," Acta Mathematica Scientia, in press.

[2] W. X. Chen, C. M. Li and B. Ou, "Classification of Solutions for an Integral Equation," Communications on Pure and Applied Mathematics, Vol. 59, No. 3, 2006, pp. 330343.

[3] L. Caffarelli, B. Gidas and J. Spruck, “Asymptotic Symmetry and Local Behavior of Semilinear Elliptic Equations with Critical Sobolev Growth," Communications on Pure and Applied Mathematics, Vol. 42, No. 3, 1989, pp. 271-297.

[4] B. Gidas and J. Spruck, "Global and Local Behaviour of Positive Solutions of Nonlinear Elliptic Equations," Communications on Pure and Applied Mathematics, Vol. 34, No. 6, 1981, pp. 525-598.

[5] S. H. Chen and G. Z. Lu, "Existence and Nonexistence of Positive Radial Solutions for a Class of Semilinear Elliptic System," Nonlinear Analysis, Vol. 38, No. 7, 1999, pp. 919-932.

[6] E. Mitidieri, "Nonexistence of Positive Solutions of Semilinear Elliptic Systems in $\mathbb{R}^{N}$, , Differential and Integral Equations, Vol. 9, No. 3, 1996, pp. 465-479.

[7] E. Lieb, "Sharp Constants in the Hardy-LittlewoodSobolev and Related Inequalities," Annals of Mathematics, Vol. 118, 1983, pp. 349-374.

[8] D. G. de Figueiredo and P. L. Felmer, "A Liouville-Type Theorem for Elliptic Systems," Annali della Scuola Normale Superiore di Pisa XXI, Vol. 21, No. 3, 1994, pp. 387-397.

[9] J. Busca and R. Manásevich, "A Liouville-Type Theorem for Lane-Emden Systems," Indiana University Mathematics Journal, Vol. 51, No. 1, 2002, pp. 37-51.

[10] Z. C. Zhang, W. M. Wang and K. T. Li, "Liouville-Type Theorems for Semilinear Elliptic Systems," Journal of Partial Differential Equations, Vol. 18, No. 4, 2005, pp. 304-310

[11] Y. Y. Li and M. Zhu, "Uniqueness Theorems through the Method of Moving Spheres," Duke Mathematical Journal, Vol. 80, No. 2, 1995, pp. 383-417.

[12] Y. Y. Li and L. Zhang, "Liouville-Type Theorems and Harnack-Type Inequalities for Semilinear Elliptic Equations," Journal d'Analyse Mathematique, Vol. 90, 2003, pp. 27-87. 
[13] W. X. Chen, C. M. Li and B. Ou, "Classification of Solutions for a System of Integral Equations," Communications in Partial Differential Equations, Vol. 30, No. 1-2, 2005, pp. 59-65.

[14] W. X. Chen, C. M. Li and B. Ou, "Qualitative Properties of Solutions for an Integral Equation," Discrete and Continuous Dynamical Systems, Vol. 12, No. 2, 2005, pp. 347-354.

[15] L. Ma and D. Z. Chen, "A Liouville Type Theorem for an Integral System," Communications on Pure and Applied Analysis, Vol. 5, No. 4, 2006, pp. 855-859.

[16] C. S. Lin, "A Classification of Solutions of a Conformally Invariant Fourth Order Equation in $\mathbb{R}^{N}$," Commentarii Mathematici Helvetici, Vol. 73, No. 2, 1998, pp. 206-231.

[17] L. A. Peletier and R. C. A. M. van der Vorst, "Existence and Non-Existence of Positive Solutions of Non-Linear Elliptic Systems and the Biharmonic Equation," Differential and Integral Equations, Vol. 5, No. 4, 1992, pp. 747767.

[18] J. Serrin and H. Zou, "The Existence of Positive Entire Solutions of Elliptic Hamitonian Systems," Communications in Partial Differential Equations, Vol. 23, No. 3-4, 1998, pp. 577-599.
[19] M. A. Souto, "A Priori Estimates and Existence of Positive Solutions of Semilinear Elliptic Systems," Differential and Integral Equations, Vol. 8, No. 5, 1995, pp. 12451258.

[20] E. M. Stein, "Singular Integral and Differentiability Properties of Functions," Princeton University Press, Princeton, 1970.

[21] Z. C. Zhang and Z. M. Guo, "Structure of Nontrivial Nonnegative Solutions of Singularly Perturbed Quasilinear Dirichlet Problems," Mathematische Nachrichten, Vol 280, No. 13-14, 2007, pp. 1620-1639.

[22] Z. C. Zhang and K. T. Li, "Spike-Layered Solutions of Singularly Perturbed Quasilinear Dirichlet Problems," Journal of Mathematical Analysis and Applications, Vol. 283, No. 2, 2003, pp. 667-680.

[23] Z. C. Zhang and K. T. Li, "Spike-Layered Solutions with Compact Support to Some Singularly Perturbed Quasilinear Elliptic Problems in General Smooth Domains," Journal of Computational and Applied Mathematics, Vol. 162, No. 2, 2004, pp. 327-340.

[24] L. P. Zhu, Z. C. Zhang and W. M. Wang, "On the Positive Solutions for a Class of Semilinear Elliptic Systems," Mathematical Applications, Vol. 19, No. 2, 2006, pp. 440445. 